\title{
Expectation maximization transfer learning and its application for bionic hand prostheses
}

\author{
Benjamin Paaßen ${ }^{1}$, Alexander Schulz ${ }^{1}$, Janne Hahne ${ }^{2}$, and Barbara Hammer ${ }^{1}$ \\ ${ }^{1}$ Center of Excellence Cognitive Interaction Technology \\ Bielefeld University \\ \{bpaassen|aschulz|bhammer\}@techfak.uni-bielefeld.de \\ ${ }^{2}$ Neurorehabilitation Systems Research Group \\ Department of Trauma Surgery \\ Orthopedic Surgery and Hand Surgery \\ Universiy Medical Center Göttingen
}

This is a preprint of a publication to appear in the Journal Neurocomputing (Special Issue regarding ESANN 2017) as provided by the authors.

\begin{abstract}
Machine learning models in practical settings are typically confronted with changes to the distribution of the incoming data. Such changes can severely affect the model performance, leading for example to misclassifications of data. This is particularly apparent in the domain of bionic hand prostheses, where machine learning models promise faster and more intuitive user interfaces, but are hindered by their lack of robustness to everyday disturbances, such as electrode shifts. One way to address changes in the data distribution is transfer learning, that is, to transfer the disturbed data to a space where the original model is applicable again. In this contribution, we propose a novel expectation maximization algorithm to learn linear transformations that maximize the likelihood of disturbed data according to the undisturbed model. We also show that this approach generalizes to discriminative models, in particular learning vector quantization models. In our evaluation on data from the bionic prostheses domain we demonstrate that our approach can learn a transformation which improves classification accuracy significantly and outperforms all tested baselines, if few data or few classes are available in the target domain.
\end{abstract}

\section{Introduction}

Classical machine learning theory relies on the assumption that training and test data stem from the same underlying distribution; an assumption, that is oftentimes violated in practical applications [6]. The reasons for such violations are multifold. The training data may be selected in a biased way and not represent the "true" distribution properly [6], 
disturbances may lead to changes in the data over time [8], or one may try to transfer an existing model to a new domain [3]. If such violations occur, the model may not accurately describe the data anymore, leading to errors, e.g. in classification.

This is particularly apparent in the domain of bionic hand prostheses. By now, research prototypes of such prostheses feature up to 20 active degrees of freedom (DoF), promising to restore precise and differentiated hand functions 2. However, controlling this many degrees of freedom requires a user interface which reacts rapidly and is intuitive to the user. A popular approach to achieve such a user interface is to let users execute the desired motion with their phantom hand, which is still represented in the brain, and infer the desired motion via classification of the residual muscle signals in the forearm, such that the desired motion can then be executed by a bionic hand prosthesis in real-time (time delay below $200 \mathrm{~ms}$ ) [10. More precisely, if a user executes a motion with her phantom hand, the corresponding neurons in the brain are activated and propagate the motor command to the arm, where the residual muscles responsible for the hand motion are activated. This activity can be recorded via a grid of electromyographic (EMG) electrodes placed on the skin around the amputee's forearm (see figure 1, top left). The EMG signal contains information about the firing pattern of the motor neurons, which in turn codes the intended hand motion. Therefore, one can classify the EMG signal with respect to the intended hand motion and use the classification result to control a prosthesis with little time delay in an intuitive way [10.

Unfortunately, such user interfaces are seriously challenged by changes in the input data distribution due to disturbances to the EMG signal, for example by electrode shifts, posture changes, sweat, fatigue, etc. [10, 14]. As an example, consider figure 1, which illustrates the effect of an electrode shift around the forearm, leading to different EMG sensor data, which in turn may cause an erroneous classification decision.

Changes between training and test distribution have been addressed by different theoretical frameworks. Shimodaira has introduced the notion of covariate shift describing the case of a change in the prior distribution $p(\vec{x})$ while the conditional distribution of the label $P(y \mid \vec{x})$ remains unchanged [25]. A slightly different angle is taken by sample selection bias correction theory which assumes that a true underlying distribution $P(y, \vec{x})$ exists from which some pairs are not available in the training data, thereby biasing the resulting machine learning model [6]. In contrast, the theory of concept drift models the prior distribution $p(\vec{x})$ and the conditional distribution $P(y \mid \vec{x})$ as varying in time. In particular, a covariate shift, meaning a change in $p(\vec{x})$ over time while $P(y \mid \vec{x})$ stays constant, is called virtual concept drift. A change in $P(y \mid \vec{x})$ over time is called real concept drift. Prior research in concept drift has focussed on either adapting a model over time to smooth and slow concept drifts or detecting a point of sudden concept drift, such that the old model can be discarded and a new model can be learned [8]. Recently, explicit long and short term memory models demonstrated an excellent ability to cope with different types of concept drift [16].

Our example of electrode shifts in bionic hand prostheses is best described by a sudden, real concept drift, in which case concept drift theory would recommend to discard the existing classifier and re-train a new one [8]. However, re-learning a viable classifier model 

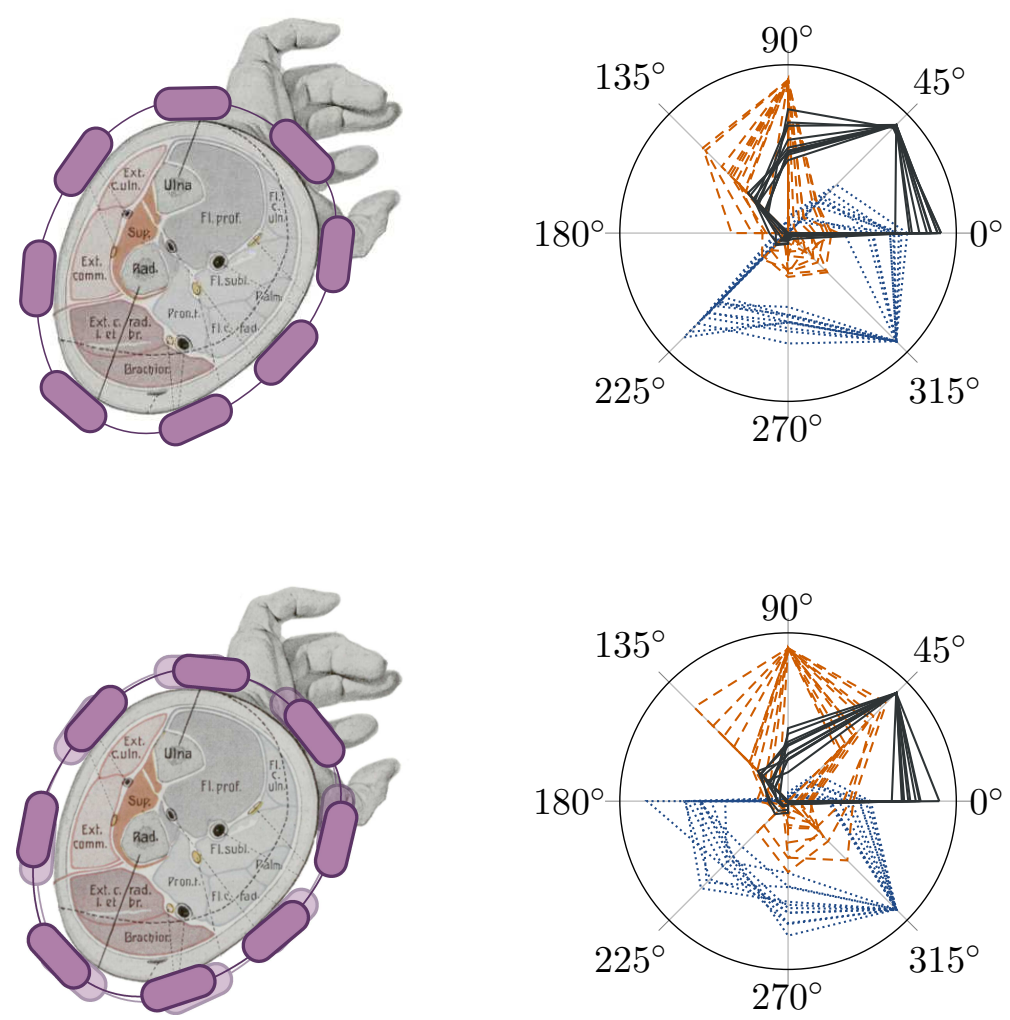

Figure 1: An illustration of electrode shifts in electromyographic (EMG) data. Top left: A grid of eight EMG electrodes placed around the forearm of a user. Cross section of the arm taken from the 1921 German edition of "Anatomie des Menschen", which is in the public domain. Top right: Example EMG signals from an eight-electrode EMG recording for two different hand motions (dashed and dotted lines) as well as resting (solid lines). Bottom left: The electrode grid is shifted around the forearm (electrode shift). Bottom right: Another set of of EMG signals from a shifted eight-electrode EMG recording for two different hand motions (dashed and dotted lines) as well as resting (solid lines). Due to the shifted signal, a model trained on the source data (top right) may misclassify shifted data (bottom right). 
may require considerable amounts of new training data to be recorded, which is inconvenient or even infeasible in user's everyday lives. Instead, we would like to re-use an existing classifier model and adapt it to the disturbed situation. This approach is motivated by prior research on myoelectric data which indicates that disturbances to electrode shifts are typically simple in structure, that is, they tend to be signal amplitude changes and shifts in the frequency spectrum [14]. Therefore, learning to transfer between the disturbed and the undisturbed setting may be considerably simpler compared to learning a new model [17.

Learning such transfers between domains has been studied in the fields of domain adaptation and transfer learning. Domain adaptation refers to re-using an existing model in another domain where little to none new training samples are available [3]. Similarly, transfer learning refers to the transfer of knowledge from a source domain, where a viable model is available, to a target domain, where the prior and/or conditional distribution is different [19]. In particular, rather than adjusting the probability distribution in a given data space, transfer learning focusses on adapting the data representation. Conceptually, this fits well to our setting as the data representation in terms of EMG readings changes, while the underlying data source, i.e. the neural code of the desired motion, remains the same.

Our key contribution is an efficient algorithm for transfer learning on labeled Gaussian mixture models relying on expectation maximization [7]. In particular, we learn a linear transformation which maps the target space training data to the source space such that the likelihood of the target space data according to the source space model is maximized. This approach generalizes to discriminative models, in particular learning vector quantization models, such as generalized matrix learning vector quantization (GMLVQ), or its localized version, LGMLVQ [22]. We evaluate our approach on artificial as well as real myoelectric data and show that our transfer learning approach can learn a transfer mapping which improves classification accuracy significantly and outperforms all tested baselines, if few samples from the target space are available and/or these samples do not cover all classes.

We begin by discussing related work, continue by introducing our own approach and conclude by evaluating our approach in comparisons to baselines from the literature.

\section{Related Work}

We begin our comparison to related work by introducing some key concepts of transfer learning more formally. In our setting, we assume that a classification model $f: \mathcal{X} \rightarrow$ $\{1, \ldots, L\}$ has been trained in some source space $\mathcal{X}=\mathbb{R}^{m}$ for some $m \in \mathbb{N}$ and we want to apply this model $f$ in some target space $\hat{\mathcal{X}}=\mathbb{R}^{n}$ for some $n \in \mathbb{N}$. Note that we assume that the classification task itself is the same for both spaces. This makes our setup an instance of domain adaptation [3] or transductive transfer learning [19]. In the example of an electrode shift on EMG data, we have $m=n$, but a simple application of our source space classifier $f$ is hindered by a fact that the activation pattern is rotated in the feature space and thus the joint distribution $p_{\hat{\mathcal{X}}}(\hat{x}, \hat{y})$ for data $\hat{x} \in \hat{\mathcal{X}}$ and labels $\hat{y} \in\{1, \ldots, L\}$ in the target space differs from the joint distribution in the source space $p_{\mathcal{X}}(\vec{x}, y)$ (see figure 

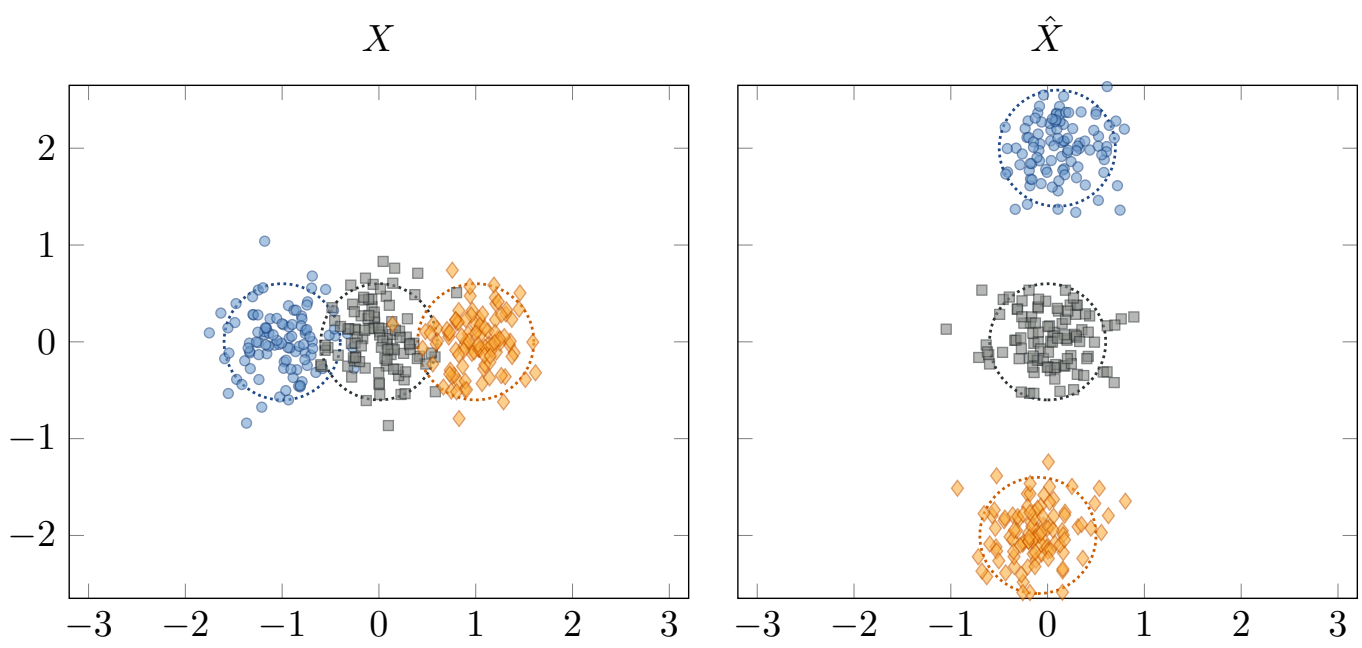

Figure 2: A simple two-dimensional data set illustrating transfer learning for a classification problem with three classes. The source space data (left) is generated by a labeled Gaussian mixture model with one component for the first (circles), the second (squares), and the third class (diamonds) respectively. The Gaussians have means $(-1,0),(0,0)$ and $(1,0)$ and standard deviation 0.3 (indicated by dashed circles at two standard deviations). The target space data (right) is generated by a similar model, but the Gaussian means are now at $(-0.1,-2),(0,0)$ and $(0.1,2)$ respectively.

1).

One family of approaches to address domain adaptation problems are importance sampling approaches, such as kernel mean matching [12], which apply a weight to each data point in the source space and re-learn the model $f$ with these weighted data points in order to generalize better to the target space [19]. The weights approximate the fraction $\frac{p_{\hat{\mathcal{X}}}(\vec{x})}{p_{\mathcal{X}}(\vec{x})}$, that is, the proportion of the probability of a point in the source space and in the target space. It can be shown that these weights minimize the empirical risk in the target space, if the conditional distributions in both spaces are equal, that is, $p_{\hat{\mathcal{X}}}(y \mid \vec{x})=p_{\mathcal{X}}(y \mid \vec{x})[19]$. However, this rather demanding assumption does not hold in our case because electrodes shift on EMG data also influence the distribution of labels.

Another family of approaches attempts to map the data from both the source and the target space to a shared latent space in which a model can be learned that applies to both the source and the target space [19, 5]. While these approaches are more general, they typically do not take label information into account, which makes transfer learning in cases such as ours significantly more challenging. Consider the simple example in figure 2, which displays a data set with three classes, where the class-specific distribution $p(\vec{x} \mid y)$ for each label $y$ is given as a Gaussian with means means $(-1,0),(0,0)$, and $(1,0)$ respectively. A classifier trained on this data set is likely to identify the $x_{1}$-axis as discriminative dimension and assign every data point with $x_{1} \leq-0.5$ to class 1 , every data point with $-0.5<x_{1}<$ 
0.5 to class 2 and any other data point to class 3 . Now, we want to transfer this model to the target space where the data representation has changed (namely, the data set is rotated and the means are moved further apart). Without any information regarding the conditional distribution of the target space data, it is inherently difficult to learn a viable transfer mapping because the assignment between the clusters in the target space and the clusters in the source space is ambiguous. In particular, a solution that maximizes the correspondence of the marginal distributions is to map the mean of the class 1 cluster to the mean of the class 3 cluster and vice versa. Conversely, if label information from the target space is available, we can disambiguate the assignment and thus simplify the transfer learning problem.

One approach which does take label information into account is the adaptive support vector machine (a-SVM) [26]. It assumes that some classifier has been trained on source space data and can assign labels to the labeled target space training data points. The a-SVM then attempts to predict the difference between the predicted labels by the source space classifier and the actual target space labels, such that it does not have to classify points which are already correctly classified by the original classifier [26]. Note that for our example in figure 2, the source model classification is only correct for the middle cluster and the a-SVM needs to re-learn the classification for the remaining two classes even though the change in representation between source and target space is structurally simple. In that sense, the a-SVM does more than is necessary in this case and does not exploit our knowledge about the task perfectly. Still, we will consider it as a baseline model in our experiments.

A recent framework which tries to explicitly learn the change in representation between source and target space is linear supervised transfer learning [17]. Assuming some classifier $f: \mathcal{X} \rightarrow\{1, \ldots, L\}$ in the source space, the approach attempts to learn a mapping $h$ : $\hat{\mathcal{X}} \rightarrow \mathcal{X}$ which minimizes the error of the classifier $f \circ h$ on the labeled target space data. In other words, the approach attempts to learn the change in representation between target and source space such that the target space data can be classified correctly by the source space classifier after mapping the data to the source space via $h$ [17. Intuitively, such an approach is particularly promising if the relationship $h$ is easier to learn compared to a new classifier $\hat{f}$ for the target space. To ensure this constraint, the authors assume that $h$ can be approximated by a linear function, that is, the first-order Taylor approximation without constant term is a good approximation of $h$ for the given data [21]. Note that the mapping $h$ needs to be optimized with respect to the model error, such that the optimization process is inherently specific to a certain source space classifier. Until now, a gradient descent scheme on the cost function of generalized learning matrix vector quantization (GMLVQ) has been suggested and shown to be effective for EMG data classification [20]. In our contribution, we extend this work in several key points. First, we provide a more precise notion of linear supervised transfer learning in a probabilistic sense. Second, we provide a general expectation maximization algorithm to optimize the model fit for labeled Gaussian mixture models. Third, we apply this algorithm to models of the learning vector quantization family, such as GMLVQ and localized GMLVQ [22], and show that we can outperform prior approaches in terms of classification accuracy on EMG data. 


\section{Transfer Learning for Labeled Gaussian Mixtures}

We begin the description of our proposed approach by re-phrasing the basic problem of transfer learning in probabilistic terms. We start with a source space $\mathcal{X}$, in which labeled data points $(\vec{x}, y)$ with $y \in\{1, \ldots, L\}$ are generated according to a joint probability density $p_{\mathcal{X}}(\vec{x}, y)$. In this space, we train a source classifier $f: \mathcal{X} \rightarrow\{1, \ldots, L\}$. Then, we wish to apply this classifier to data in a target space $\hat{\mathcal{X}}=\mathbb{R}^{n}$ for some $n \in \mathbb{N}$, where labeled data points $(\hat{x}, \hat{y})$ are generated according to the probability density $p_{\hat{\mathcal{X}}}(\hat{x}, \hat{y})$ with $\hat{y} \in$ $\{1, \ldots, L\}$. Our basic assumption is that there exists a smooth function $h: \hat{\mathcal{X}} \rightarrow \mathcal{X}$ such that for all $\hat{x} \in \hat{\mathcal{X}}$ and all $\hat{y} \in\{1, \ldots, L\}$ it holds: $p_{\hat{\mathcal{X}}}(\hat{x}, \hat{y})=p_{\mathcal{X}}(h(\hat{x}), \hat{y})$. In other words: We assume, that there is a smooth mapping $h$ that characterizes the change in representation between target and source space and therefore fully explains the differences in the joint probability densities. We propose to learn this mapping $h$ using a maximum likelihood approach. In particular, we intend to construct a model of the joint probability density for data and labels in the source space $p(\vec{x}, y)$. Then, we intend to maximize the empiric likelihood of a labeled example data set from the target space $\left\{\left(\hat{x}_{j}, \hat{y}_{j}\right)\right\}_{j \in\{1, \ldots, N\}}$, according to the source space model, that is, we attempt to solve the optimization problem

$$
\max _{h} \prod_{j=1}^{N} p\left(h\left(\hat{x}_{j}\right), \hat{y}_{j}\right)
$$

Note that, if we only care about classification, we do not require $p(\vec{x}, y)$ to be a precise, generative model for the source space data. In this case, it suffices if we have a precise model of $P(y \mid \vec{x})$ as provided by many classifiers, whereas the approximation of the data density $p(\vec{x})$ may be inaccurate. Therefore, we disregard the specific difficulties of inferring a precise density model within this contribution.

In the remainder of this work, we will provide a solution for the maximization problem 1 for a special class of models, namely Gaussian mixture models for $p(\vec{x}, y)$ and linear functions $h$.

\subsection{Labeled Gaussian Mixture Models}

First, we choose to approximate $p_{\mathcal{X}}(\vec{x}, y)$ with a Gaussian mixture model (GMM). Such models are well-established in machine learning [4, 1] and have been successfully applied to classify EMG data [13. In general, almost any density can be approximated via a GMM, if a sufficient number of Gaussian components $K$ is employed [4]. GMMs approximate a density $p(\vec{x})$ via a sum of Gaussians $k=1, \ldots, K$ as follows [4, 1]:

$$
\begin{aligned}
& p(\vec{x}) \approx \sum_{k=1}^{K} \mathcal{N}\left(\vec{x} \mid \vec{\mu}_{k}, \Lambda_{k}\right) \cdot P(k) \quad \text { where } \\
& \mathcal{N}\left(\vec{x} \mid \vec{\mu}_{k}, \Lambda_{k}\right)=\sqrt{\frac{\operatorname{det}\left(\Lambda_{k}\right)}{(2 \cdot \pi)^{m}}} \cdot \exp \left(-\frac{1}{2} \cdot\left(\vec{x}-\vec{\mu}_{k}\right)^{T} \cdot \Lambda_{k} \cdot\left(\vec{x}-\vec{\mu}_{k}\right)\right)
\end{aligned}
$$


where $\vec{\mu}_{k}$ is the mean of the $k$ th Gaussian and $\Lambda_{k}$ is the precision matrix (a positive definite matrix, which is the inverse of the covariance matrix) of the $k$ th Gaussian.

In our setting, we intend to apply Gaussian mixture models for classification, which means that we need to include the label $y$ of the data in the model. In particular, we re-write the joint probability density $p(\vec{x}, y)$ as follows:

$$
p(\vec{x}, y)=\sum_{k=1}^{K} p(\vec{x}, y, k)=\sum_{k=1}^{K} p(\vec{x} \mid y, k) \cdot P(y \mid k) \cdot P(k)
$$

As before we assume that $p(\vec{x} \mid k)$ is a Gaussian density. Additionally, we assume that the label $y$ of a data point and the data point $\vec{x}$ itself are conditionally independent given the Gaussian component $k$ they have been generated from. Under these assumptions we obtain:

$$
p(\vec{x}, y)=\sum_{k=1}^{K} \mathcal{N}\left(\vec{x} \mid \vec{\mu}_{k}, \Lambda_{k}\right) \cdot P(y \mid k) \cdot P(k)
$$

The parameters of our model are, for each Gaussian component $k$, a mean $\vec{\mu}_{k}$, a precision matrix $\Lambda_{k}$, a probability distribution over the labels $P(y \mid k)$ and a prior probability $P(k)$. A simple example of such a model is shown in figure 2 (left). The parameters for this labeled Gaussian mixture model (lGMM) are $\vec{\mu}_{1}=(-1,0), \vec{\mu}_{2}=(0,0)$, and $\vec{\mu}_{3}=(1,0)$ as means, $\Lambda_{1}=\Lambda_{2}=\Lambda_{3}=\frac{1}{0.3^{2}} \cdot I^{m}$ as precision matrices, where $I^{m}$ is the $m \times m$-dimensional identity matrix, and $P(y \mid k)=1$ if $y=k$ and 0 otherwise, as well as $P(k)=\frac{1}{3}$ for all $k$.

We can classify data with an lGMM via a maximum a posteriori approach, where the posterior is:

$$
P(y \mid \vec{x})=\frac{p(\vec{x}, y)}{p(\vec{x})}=\frac{\sum_{k=1}^{K} \mathcal{N}\left(\vec{x} \mid \vec{\mu}_{k}, \Lambda_{k}\right) \cdot P(y \mid k) \cdot P(k)}{\sum_{k=1}^{K} \mathcal{N}\left(\vec{x} \mid \vec{\mu}_{k}, \Lambda_{k}\right) \cdot P(k)}
$$

We now turn to the question how to learn a transfer function which maximizes the likelihood of target space data according to an lGMM in the source space.

\subsection{Linear supervised transfer learning via expectation maximization}

Our second approximation to make the transfer learning problem 1 feasible is to approximate the transfer function $h$ with a linear function. Equivalently, we could say that there exists a matrix $H \in \mathbb{R}^{m \times n}$ such that for all $\hat{x}_{j}$ in our target space data it holds: $h\left(\hat{x}_{j}\right) \approx H \cdot \hat{x}_{j}$. Another perspective is provided by Saralajew and Villmann, who frame linear supervised transfer learning as trying to find the first-order Taylor expansion of $h$, while ignoring the constant term [21. The linearity restriction may appear rather harsh, but it does have a justification: Transfer learning is only viable if $h$ is simple compared to the classifier $f$. Otherwise one could simply dismiss the source space model whenever a change in representation occurs and learn a new model for the target space, as most drift detection approaches do [8]. Our linearity restriction ensures that $h$ has a simple form and is therefore simple to learn [17]. Another advantage of the linearity restriction is that 
the function $h$ can be parametrized by a single matrix $H$. More precisely, we obtain the following new form for 1, after applying the logarithm:

$$
\max _{H} \sum_{j=1}^{N} \log \left[\sum_{k=1}^{K} \mathcal{N}\left(H \cdot \hat{x}_{j} \mid \vec{\mu}_{k}, \Lambda_{k}\right) \cdot P\left(y_{j} \mid k\right) \cdot P(k)\right]
$$

Note that even this restricted problem is challenging. Consider the example in figure 3 (top). The data is generated with an lGMM with the same parameters as in figure 2. except that the label distribution $P(y \mid k)$ is now given as $P(1 \mid 1)=P(2 \mid 2)=P(1 \mid 3)=1$ and 0 otherwise, implying that the label 3 is not generated anymore. In this case, the transfer mapping $h$ could either rotate the target data $\hat{X}$ to the left or to the right, resulting in the same likelihood (see figure 3 , bottom left). In contrast, for the non-ambiguous labeling in figure 2, this problem does not occur. In this case, the likelihood has a single global optimum (see figure 3, bottom right). This illustrates how label information contributes crucial disambiguating information for transfer learning.

The problem of finding a (local) optimum for $H$ with respect to the log-likelihood in equation 7 can be addressed using an expectation maximization (EM) scheme, as proposed by Dempster, Laird and Rubin [7]. Note that we refer to EM here as a general optimization scheme for parameters under latent variables, not as a specific optimization scheme for Gaussian mixture models as discussed by [4, 1. In particular, we do not intend to adapt the means and covariance matrices of the Gaussian mixture model in our transfer learning scheme. Instead, we only adapt the transfer matrix $H$, which makes the structure of the problem considerably simpler.

The general EM scheme has two steps, an expectation step and a maximization step. In the expectation step, we compute the posterior for the latent variables given the current parameters and in the maximization step we set the parameters in order to maximize the expected $\log$ likelihood $Q$ with respect to the latent variables. The EM scheme starts with some initial value for the parameters and then iterates the two steps until $Q$ converges. Dempster, Laird and Rubin have shown that this scheme is guaranteed to achieve a local optimum for the actual log likelihood [7].

In our case, we intend to optimize the transfer matrix $H$, treating the assignment of data points to Gaussian components as latent variables. Thus, our expectation step is to compute the posterior for $k \in\{1, \ldots, K\}$ with respect to every transferred test data point $\left(H \cdot \hat{x}_{j}, \hat{y}_{j}\right)$, while keeping $H$ fixed. For compactness, we denote the posterior $P\left(k \mid H \cdot \hat{x}_{j}, \hat{y}_{j}\right)$ as $\gamma_{k \mid j}$. We obtain:

$$
\begin{aligned}
\gamma_{k \mid j} & :=P\left(k \mid H \cdot \hat{x}_{j}, \hat{y}_{j}\right)=\frac{p\left(H \cdot \hat{x}_{j}, \hat{y}_{j} \mid k\right) \cdot P(k)}{p\left(H \cdot \hat{x}_{j}, \hat{y}_{j}\right)} \\
& =\frac{p\left(H \cdot \hat{x}_{j} \mid k\right) \cdot P\left(\hat{y}_{j} \mid k\right) \cdot P(k)}{\sum_{k^{\prime}=1}^{K} p\left(H \cdot \hat{x}_{j} \mid k^{\prime}\right) \cdot P\left(\hat{y}_{j} \mid k^{\prime}\right) \cdot P\left(k^{\prime}\right)}
\end{aligned}
$$

Note that $\gamma_{k \mid j}$ can degenerate if a precision matrix $\Lambda_{k}$ is not full rank. In such cases, the determinant $\operatorname{det}\left(\Lambda_{k}\right)$ is 0 and thus $\mathcal{N}\left(\vec{x} \mid \vec{\mu}_{k}, \Lambda_{k}\right)=0$ is not a valid density. Such degenerations can be prevented by ensuring that all Eigenvalues of $\Lambda_{k}$ stay above some 

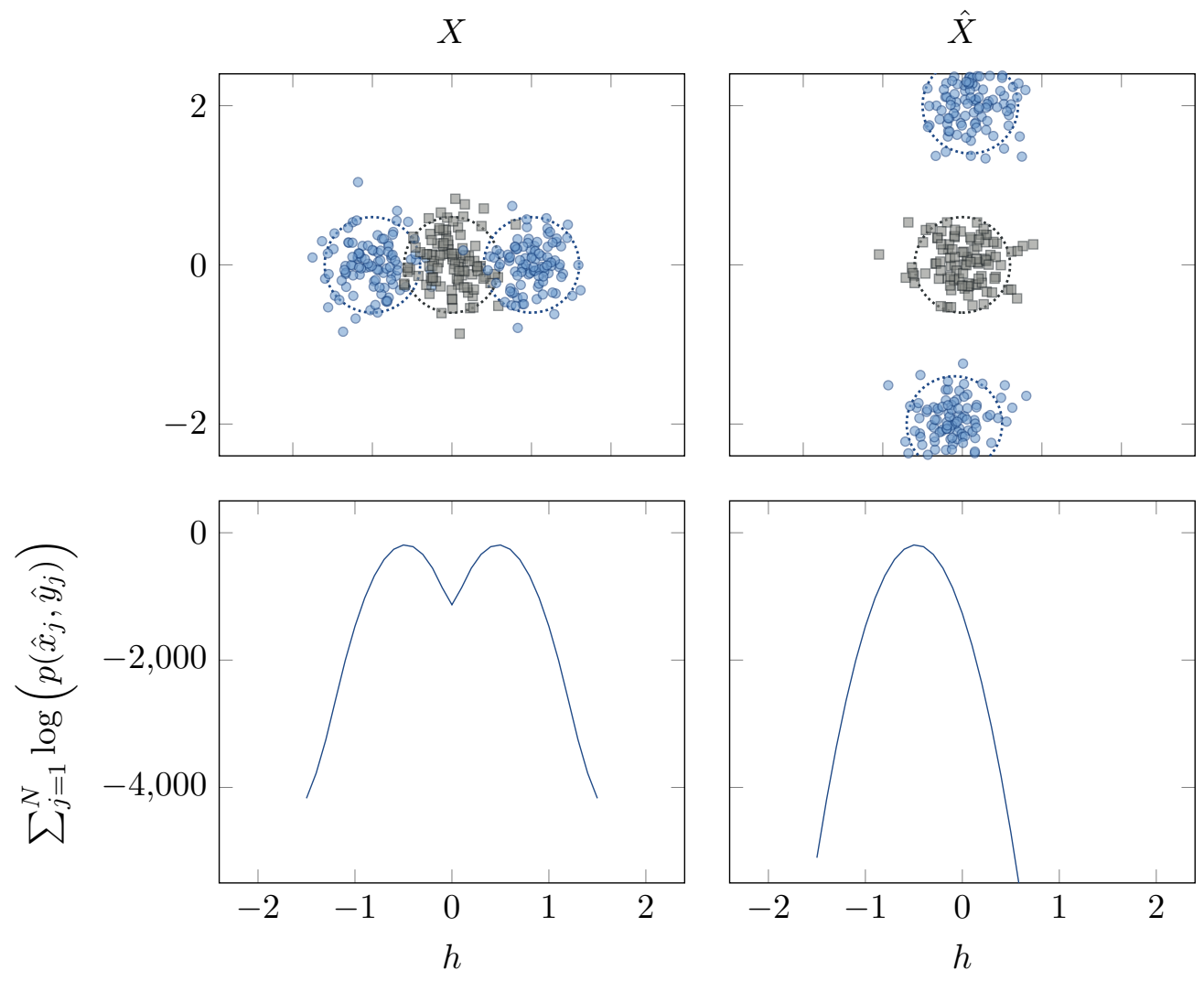

Figure 3: Top left and right: A transfer learning problem with an ambiguous transfer mapping. The data has been generated as in figure 2 with the difference that the data clusters to the left and right in the left figure and the top and bottom in the right figure share the same label. Bottom left: The log-likelihood according to equation 7 of transfer mappings of the form $H=(0, h ; 0,0)$. $h$ is shown on the x-axis, while the log-likelihood is shown on the y-axis. As can be seen, the log-likelihood has two local optima. Bottom right: The log-likelihood if the generating model is constructed as in figure 2 . In this case, there is only one global optimum. 
minimum value [4, 1] or by replacing the determinant by the pseudo-determinant, which is defined as the product of all non-zero Eigenvalues. The former strategy assigns some relevance to all dimensions of the data space and thus is better able to identify outliers, while the latter strategy entirely disregards dimensions in which no data variance occurs. Subsequently, we will generally assume that one of these two strategies is applied to treat degenerate densities.

In the maximization step, we keep the posterior values $\gamma_{k \mid j}$ fixed and adapt $H$ in order to adapt the expected $\log$ likelihood $Q$ with respect to the latent variables. We denote the adapted transfer matrix as $H$ and the transfer matrix from the previous iteration as $H^{\text {old }}$. Then, we obtain for the maximization step:

$$
\begin{aligned}
\max _{H} Q\left(H \mid H^{\text {old }}\right) & =\max _{H} \sum_{j=1}^{N} \mathbb{E}_{k \mid H^{\text {old }} \cdot \hat{x}_{j}, \hat{y}_{j}}\left[\log \left(p\left(H \cdot \hat{x}_{j}, \hat{y}_{j}, k\right)\right)\right] \\
& =\max _{H} \sum_{j=1}^{N} \sum_{k=1}^{K} \gamma_{k \mid j} \cdot \log \left(p\left(H \cdot \hat{x}_{j}, \hat{y}_{j}, k\right)\right) \\
& =\max _{H} \sum_{j=1}^{N} \sum_{k=1}^{K} \gamma_{k \mid j} \cdot\left(\log \left[p\left(H \cdot \hat{x}_{j} \mid k\right)\right]+\log \left[P\left(\hat{y}_{j} \mid k\right)\right]+\log [P(k)]\right)
\end{aligned}
$$

Note that neither $P\left(\hat{y}_{j} \mid k\right)$ nor $P(k)$ depend on $H$, such that we can disregard them for our optimization problem. If we further plug in equation 3 we obtain:

$$
\begin{aligned}
& \max _{H} \sum_{j=1}^{N} \sum_{k=1}^{K} \gamma_{k \mid j} \cdot \log \left[\sqrt{\frac{\operatorname{det}\left(\Lambda_{k}\right)}{(2 \cdot \pi)^{m}}} \cdot \exp \left(-\frac{1}{2} \cdot\left(H \cdot \hat{x}-\vec{\mu}_{k}\right)^{T} \cdot \Lambda_{k} \cdot\left(H \cdot \hat{x}-\vec{\mu}_{k}\right)\right)\right] \\
= & \min _{H} \sum_{j=1}^{N} \sum_{k=1}^{K} \gamma_{k \mid j} \cdot\left(H \cdot \hat{x}_{j}-\vec{\mu}_{k}\right)^{T} \cdot \Lambda_{k} \cdot\left(H \cdot \hat{x}_{j}-\vec{\mu}_{k}\right)=: E_{Q}(H)
\end{aligned}
$$

Note that this is just a weighted quadratic error between Gaussian means $\vec{\mu}_{k}$ and data points $H \cdot \hat{x}_{j}$. We denote this quantity as $E_{Q}(H)$. Interestingly, in this form, the optimization problem becomes convex and therefore has a guaranteed global solution.

Theorem 1. $E_{Q}$ is a convex function. Further, the gradient of $E_{Q}$ is given as:

$$
\nabla_{H} E_{Q}(H)=2 \cdot \sum_{k=1}^{K} \Lambda_{k} \cdot \sum_{j=1}^{N} \gamma_{k \mid j} \cdot\left(H \cdot \hat{x}_{j}-\vec{\mu}_{k}\right) \cdot \hat{x}_{j}^{T}
$$

Proof. To define the gradient of a function with respect to a matrix we consider the derivative with respect to all matrix entries and put these back into a matrix of the original form. Via this mechanism, we obtain the gradient

$$
\nabla_{H}\left(H \cdot \hat{x}_{j}-\vec{\mu}_{k}\right)^{T} \cdot \Lambda_{k} \cdot\left(H \cdot \hat{x}_{j}-\vec{\mu}_{k}\right)=2 \cdot \Lambda_{k} \cdot\left(H \cdot \hat{x}_{j}-\vec{\mu}_{k}\right) \cdot \hat{x}_{j}^{T}
$$

which leads straightforwardly to equation 12 . 
For the convexity proof we inspect the Hessian of $E_{Q}(H)$. Here, we define the Hessian as the matrix of second second derivatives with respect to all entries of $H$. This Hessian is given as [9]:

$$
\nabla_{H}^{2} E_{Q}(H)=2 \cdot \sum_{k=1}^{K} \Lambda_{k} \otimes\left(\sum_{j=1}^{N} \gamma_{k \mid j} \cdot \hat{x}_{j} \cdot \hat{x}_{j}^{T}\right)
$$

where $\otimes$ is the Kronecker product of two matrices. Recall that $\Lambda_{k}$ is a positive definite matrix and note that $\sum_{j=1}^{N} \gamma_{k \mid j} \cdot \hat{x}_{j} \cdot \hat{x}_{j}^{T}$ is a positive (semi-)definite matrix. Further, the Kronecker product of positive (semi-)definite matrices, as well as the sum of positive (semi-)definite matrices is also guaranteed to be positive (semi-)definite [9]. Therefore, the Hessian of $E_{Q}(H)$ is positive (semi-)definite, which shows that $E_{Q}$ is convex.

Therefore, we can find a global optimum of $E_{Q}$ efficiently, using some gradient-based solver, such as the limited memory Broyden-Fletcher-Goldfarb-Shanno algorithm (l-BFGS). An even more efficient optimization is possible if all Gaussian components share the same covariance matrix. In this case, we can provide an analytic solution to 11.

Theorem 2. Let $\hat{X}=\left(\hat{x}_{1}, \ldots, \hat{x}_{N}\right) \in \mathbb{R}^{n \times N}, \Gamma \in \mathbb{R}^{K \times N}$ with $\Gamma_{k j}=\gamma_{k \mid j}$, and $W=$ $\left(\vec{\mu}_{1}, \ldots, \vec{\mu}_{K}\right) \in \mathbb{R}^{m \times K}$.

If the matrix $\hat{X} \cdot \hat{X}^{T}$ is full rank and there is a matrix $\Lambda \in \mathbb{R}^{m \times m}$, such that for all $k \in\{1, \ldots, K\}$ it holds $\Lambda_{k}=\Lambda$, then $E_{Q}(H)$ has a global optimum at

$$
H=W \cdot \Gamma \cdot \hat{X}^{\dagger} .
$$

where $\hat{X}^{\dagger}=\hat{X}^{T} \cdot\left(\hat{X} \cdot \hat{X}^{T}\right)^{-1}$ is the pseudo-Inverse of $\hat{X}$.

Proof. As we have shown above, $E_{Q}(H)$ is convex, such that it is sufficient to find a point $H$ with $\nabla_{H} E_{Q}(H)=0$ for a global optimum. Such a point can be obtained as follows, starting from equation 12 .

$$
\begin{aligned}
2 \cdot \sum_{k=1}^{K} \Lambda \cdot \sum_{j=1}^{N} \gamma_{k \mid j} \cdot\left(H \cdot \hat{x}_{j}-\vec{\mu}_{k}\right) \cdot \hat{x}_{j}^{T} & =0 \\
\Lambda \cdot H \cdot \sum_{j=1}^{N} \hat{x}_{j} \cdot \hat{x}_{j}^{T} & =\Lambda \cdot \sum_{k=1}^{K} \sum_{j=1}^{N} \gamma_{k \mid j} \cdot \vec{\mu}_{k} \cdot \hat{x}_{j}^{T} \\
\Lambda \cdot H \cdot \hat{X} \cdot \hat{X}^{T} & =\Lambda \cdot W \cdot \Gamma \cdot \hat{X}^{T} \\
H & =W \cdot \Gamma \cdot \hat{X}^{\dagger}
\end{aligned}
$$

Note that we can avoid the problem of a rank-deficient matrix $\hat{X} \cdot \hat{X}^{T}$ by adding a small positive constant $\lambda$ to the diagonal, which ensures full rank and corresponds to adding the regularization term $\lambda \cdot \operatorname{trace}\left(\Lambda \cdot H \cdot H^{T}\right)$ to the error $E_{Q}(H)$ in equation 11 . Note the striking similarity to the Gaussian prior for linear regression [4]. 
Further note the $\Leftarrow$ on the last line of the proof. The implication is uni-directional for this step because the equation $\nabla_{H} E_{Q}(H)=0$ may have infinitely many solutions if $\Lambda$ is not full rank. In this case, we could add an arbitrary matrix to $H$ which is constructed from vectors in the null-space of $\Lambda$. Such matrices $H^{\prime}$ would still be globally optimal solutions. Equation 15 provides us with just one of those solutions.

The overall expectation maximization algorithm is displayed in algorithm 1 .

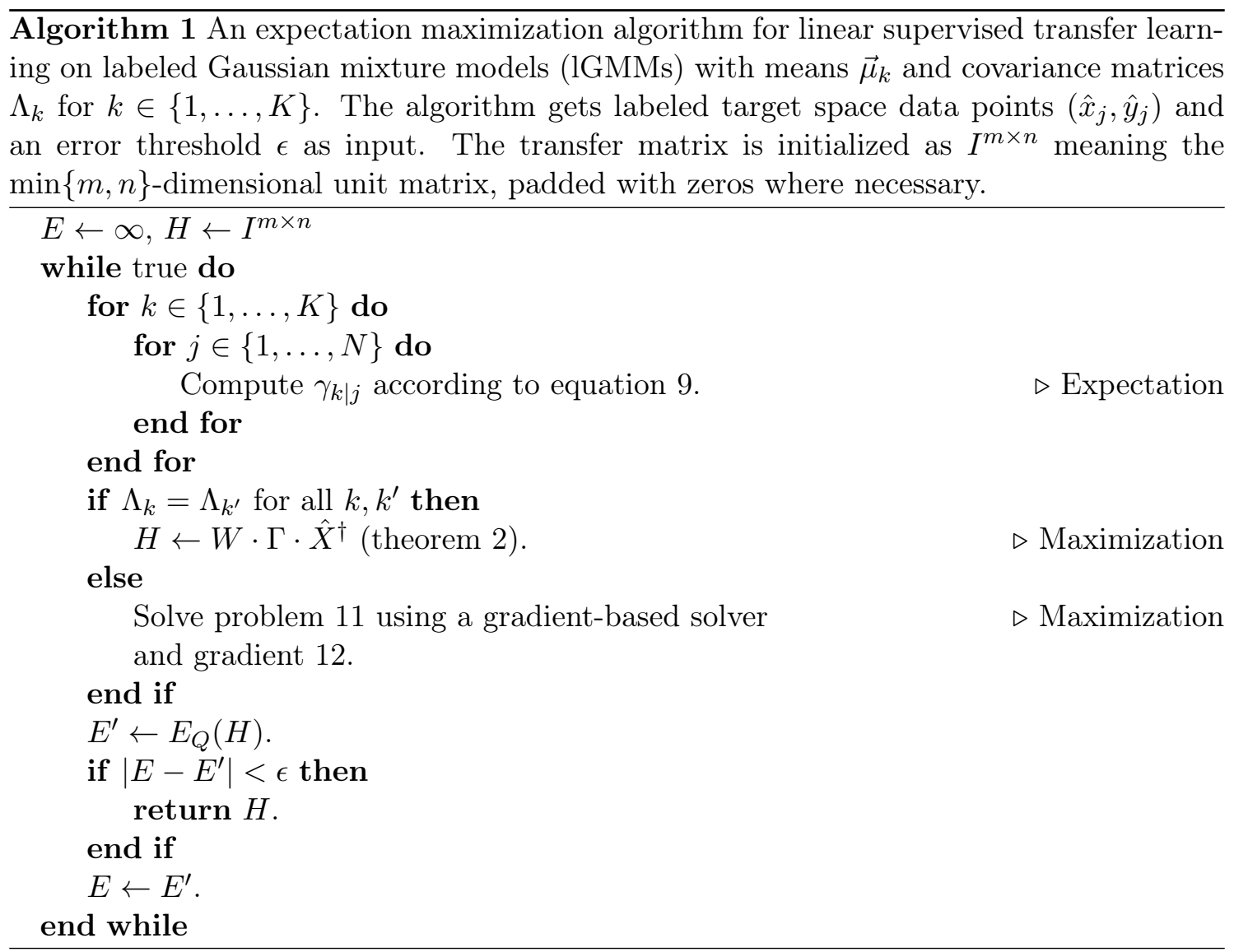

With regards to computational complexity, we analyze the expectation step and the maximization step separately. The expectation step requires the computation of $N \cdot K$ values of the likelihood $p\left(H \cdot \hat{x}_{j} \mid k\right)$ according to equation 9. Each of these computations is possible in constant time, if we treat the number of dimensions $n$ and $m$ as constants. For the maximization step, we need to consider two different cases. If $\Lambda_{k}=\Lambda_{k^{\prime}}$ for all $k, k^{\prime}$, we require $\mathcal{O}(N \cdot K)$ computations for the matrix product $W \cdot \Gamma \cdot \hat{X}^{\dagger}$ according to theorem 2. Otherwise, we require an unconstrained optimization algorithm to solve the convex problem 11. Such an algorithm will typically need to compute gradients according to equation 12 , which takes $\mathcal{O}(N \cdot K)$ computations each time. As the convex optimization problem 11 is just quadratic in nature, we will assume that a viable optimization algorithm can solve it in a constant number of gradient computations. Therefore, we obtain $\mathcal{O}(N \cdot K$. 
$T$ ) for the computational complexity of algorithm 1, where $T$ is the number of iterations it takes until the error does not change more than $\epsilon$ anymore. It is relatively simple to show that $T$ must be finite (for $\epsilon>0$ ) because the expectation maximization scheme never decreases the likelihood [1] and the likelihood is bounded by 1 . In our experimental evaluation, we find that $T$ is typically rather small (less than 30 iterations) and that the EM approach is therefore considerably faster compared to the alternatives of learning a new lGMM model or learning a transfer function on the non-convex GLVQ cost function. However, providing a theory-grounded estimate for $T$ is challenging. Still, we can gain some insight by analyzing the special case of a one-to-one assignment of components to labels, meaning that for each label $y$ there exists exactly one component $k$ such that $P(y \mid k)=1$. In this case, $\gamma_{k \mid j}$ is independent of $H$ because data points are always assigned crisply to the component with the matching label. Therefore, the first maximization step directly identifies the global maximum and in the second iteration the error will not change anymore, yielding $T=2$ (as is the case in the data from figure 2). As a rule of thumb, $T$ increases with the ambiguity in the assignment of data points to components. If there are many components which can generate a data point, finding a good assignment may take many iterations. Therefore, it is beneficial in terms of runtime to use as little components as possible, as well as "crisp" label distributions, ideally $P(y \mid k)=1$ for some label $y$. The latter point motivates the use of models of the learning vector quantization family, which feature such crisp assignments.

\subsection{EM Transfer Learning for Learning Vector Quantization Models}

If we already have trained a classifier $f$ in the source space, training an additional labeled Gaussian mixture model (lGMM) for transfer learning may appear as unnecessary overhead. Fortunately, it is possible to obtain a viable lGMM from an existing classifier model. In particular, an lGMM can be obtained based on a learning vector quantization model.

Learning vector quantization (LVQ) models describe data in terms of prototypes $\vec{\mu}_{k} \in \mathcal{X}$ which are assigned to a label $\bar{y}_{k} \in\{1, \ldots, L\}$. Data points are classified by assigning the label of the closest prototype, that is: $f(\vec{x})=\operatorname{argmin}_{y} \min _{k: \bar{y}_{k}=y} d\left(\vec{x}, \vec{\mu}_{k}\right)$ where $d$ is the Euclidean distance [15]. A probabilistic variant of LVQ is robust soft learning vector quantization (RSLVQ) which connects LVQ with Gaussian mixture models [24. RSLVQ essentially formulates a labeled Gaussian mixture model where each component generates only one label, that is, $P(y \mid k)=1$ for one label $y=\bar{y}_{k}$ and 0 otherwise. Also, RSLVQ assumes radial precision matrices $\Lambda_{k}=\frac{1}{\sigma_{k}^{2}} I^{m}$ for some positive scalar $\sigma_{k}$. The positions of the means $\vec{\mu}_{k}$ are adjusted according to a stochastic gradient ascent on the log-posterior $\log (P(y \mid \vec{x}))=\log [p(\vec{x}, y) / P(y)]$. Seo and Obermayer point out that the posterior $P(y \mid \vec{x})$ for such a model becomes a crisp winner-takes-all rule if $\sigma_{k}$ becomes small for all $k$ and therefore an RSLVQ becomes a classic LVQ model with prototypes $\vec{\mu}_{k}$ [24]. Schneider has extended the standard RSLVQ scheme to also permit and learn full precision matrices $\Lambda_{k}$, yielding a full labeled Gaussian mixture model [23].

However, even if the prototypes and precision matrices have not been learned in a probabilistically motivated fashion, we can use the connection between LVQ models and lGMM 
models for the purpose of transfer learning. As an example, consider a local generalized matrix learning vector quantization (LGMLVQ) model [22]. Such a model is trained via a stochastic gradient descent on the cost function

$$
E=\sum_{i=1}^{M} \Phi\left(\frac{d^{2}\left(\vec{\mu}^{+}, \vec{x}_{i}\right)-d^{2}\left(\vec{\mu}^{-}, \vec{x}_{i}\right)}{d^{2}\left(\vec{\mu}^{+}, \vec{x}_{i}\right)-d^{2}\left(\vec{\mu}^{-}, \vec{x}_{i}\right)}\right)
$$

where $\vec{\mu}^{+}$is the closest prototype to $\vec{x}_{i}$ with the same label, $\vec{\mu}^{-}$is the closest prototype to $\vec{x}_{i}$ with a different label, and $\Phi$ is some sigmoid function. Importantly, the training does not only adjust the position of the prototypes $\vec{\mu}_{k}$ but also adapts the distance $d^{2}\left(\vec{\mu}_{k}, \vec{x}_{i}\right)=$ $\left(\vec{\mu}_{k}-\vec{x}_{i}\right)^{T} \cdot \Omega_{k}^{T} \cdot \Omega_{k} \cdot\left(\vec{\mu}_{k}-\vec{x}_{i}\right)$ and learning the matrix $\Omega_{k}$ [22]. A notable special case of LGMLVQ is GMLVQ, in which all prototype share the same matrix $\Omega$ [22]. Also note that LGMLVQ includes generalized learning vector quantization (GLVQ) as a special case by restricting $\Omega_{k}$ to the identity matrix.

Assume now that we have trained a LGMLVQ model in the source space with prototypes $\vec{\mu}_{1}, \ldots, \vec{\mu}_{K}$ with labels $\bar{y}_{1}, \ldots, \bar{y}_{K}$ and matrices $\Omega_{1}, \ldots, \Omega_{K}$. Then, we obtain an IGMM from this model by setting the Gaussian means to the prototypes, the precision matrices to $\Lambda_{k}=\frac{1}{\sigma^{2}} \Omega_{k}^{T} \cdot \Omega_{k}$, which is guaranteed to be symmetric and positive semidefinite for any positive scalar $\sigma$. Further, as in RSLVQ, we set $P(y \mid k)=1$ if $y=\bar{y}_{k}$ and 0 otherwise [24]. Finally, we set $P(k)=\frac{1}{K}$ for all $k$.

As in RSLVQ, we can argue that the resulting lGMM classifies data points in the same way as the underlying LGMLVQ model and is therefore consistent with it. This is because the posterior in equation 6 collapses to:

$$
P(y \mid \vec{x})=\frac{\sum_{k: \bar{y}_{k}=y} p(\vec{x} \mid k)}{\sum_{k=1}^{K} p(\vec{x} \mid k)}=\frac{\sum_{k: \bar{y}_{k}=y} \sqrt{\operatorname{det}\left(\Lambda_{k}\right)} \cdot \exp \left(-\frac{1}{2 \sigma^{2}} \cdot d^{2}\left(\vec{\mu}_{k}, \vec{x}\right)\right)}{\sum_{k=1}^{K} \sqrt{\operatorname{det}\left(\Lambda_{k}\right)} \cdot \exp \left(-\frac{1}{2 \sigma^{2}} \cdot d^{2}\left(\vec{\mu}_{k}, \vec{x}\right)\right)}
$$

Let now $d_{k}:=d^{2}\left(\vec{\mu}_{k}, \vec{x}\right)$ and assume that $\vec{\mu}_{k^{+}}$is the closest prototype to $\vec{x}$, that is, $d_{k^{+}}<d_{k}$ for all $k \neq k^{+}$. By multiplying both enumerator and denominator with $\exp \left(\frac{1}{2 \sigma^{2}} d_{k^{+}}\right)$we obtain:

$$
P\left(\bar{y}_{k^{+}} \mid \vec{x}\right)=\frac{\sqrt{\operatorname{det}\left(\Lambda_{k^{+}}\right)}+\sum_{\substack{k \neq k^{+} \\ \bar{y}_{k}=\bar{y}_{k^{+}}}} \sqrt{\operatorname{det}\left(\Lambda_{k}\right)} \cdot \exp \left(-\frac{1}{2 \sigma^{2}} \cdot\left(d_{k}-d_{k^{+}}\right)\right)}{\sqrt{\operatorname{det}\left(\Lambda_{k^{+}}\right)}+\sum_{k \neq k^{+}} \sqrt{\operatorname{det}\left(\Lambda_{k}\right)} \cdot \exp \left(-\frac{1}{2 \sigma^{2}} \cdot\left(d_{k}-d_{k^{+}}\right)\right)}
$$

where $d_{k}-d_{k^{+}}>0$ for all $k \neq k^{+}$. Therefore, $\exp \left(-\frac{1}{2 \sigma^{2}} \cdot\left(d_{k}-d_{k^{+}}\right)\right)$exponentially approaches 0 for smaller $\sigma$, which leads us to $P\left(\bar{y}_{k^{+}} \mid \vec{x}\right) \approx 1$. In other words, the maximum a posteriori estimate of the label for $\vec{x}$ is to assign the label of the closest prototype, which is equivalent to the classification by LGMLVQ.

\section{Experiments}

In this section, we validate our proposed transfer learning scheme experimentally on three data sets, two artificial ones and one from the domain of bionic hand prosthesis control. For 
each data set, we train a generalized matrix learning vector quantization model (GMLVQ) or a local GMLVQ (LGMLVQ) model on the source space data. Then, we try to apply the source space model to target space data via our proposed expectation maximization (EM) transfer learning algorithm. We compare the mean classification error obtained by EM with the following reference methods:

- naively applying the source space model to the target data (naive),

- re-training a new model solely on the target space data (retrain),

- supervised linear transfer learning based on the GLVQ cost function [17], which learns a linear mapping between target and source data using stochastic gradient descent on the GLVQ cost function 20 ( GMLVQ), and

- utilizing the adaptive support vector machine ([26]), which tries to apply the source space classifier to the target space data but corrects wrong predictions using a support vector machine $(a-S V M)$.

All implementations are available in our toolbox [18].

We analyze the ability for transfer learning of all methods in different conditions: First, we vary the number of target space training data points available for transfer learning. Our hypothesis is that our proposed EM transfer learning scheme should require less data to achieve a good classification error compared to re-training a new model because it only needs to learn a simple linear transformation, compared to a potentially complex, nonlinear classification model (H1).

Second, we remove an entire class from the target space training data. This is particularly interesting in domains where recording an additional class implies significant additional effort. We hypothesize that our proposed EM transfer learning scheme should be less affected by missing classes compared to all other transfer learning approaches (H2) because its focus lies on a change in representation, not in constructing a model of the class distribution itself. If the representation change can be estimated using a subset of the classes, any class not contained in that subset should be omissible without negative effect.

For each experimental condition we report the mean classification test error on the target space data in a crossvalidation. Finally, we report the runtime of all transfer learning approaches running on a Intel Core i7-7700 HQ CPU. We expect that our proposed transfer learning approach will be considerably faster compared to re-training a new model, the aSVM and GMLVQ transfer learning (H3), because it involves only a convex optimization for a linear transformation matrix with fairly few parameters.

For all significance tests we employ a one-sided Wilcoxon signed rank test.

\subsection{Artificial Data I}

Our first data set is the two-dimensional toy data set shown in figure 2 The data is generated via a labeled Gaussian mixture model with one component for each of the three 

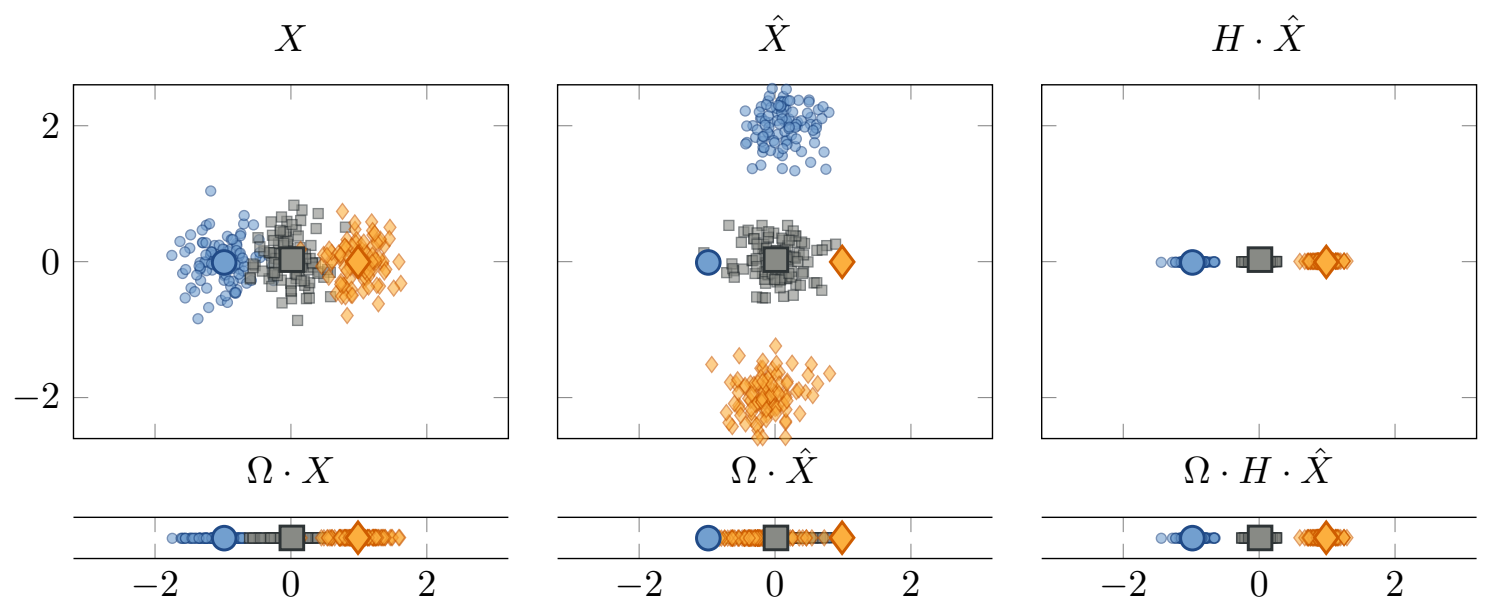

Figure 4: A visualization of a GMLVQ model trained on the toy data set shown in figure2. Prototypes are highlighted via bigger size. Shape and color indicate the class assignment. Top row: The source space data $X$, target space data $\hat{X}$ and transferred target space data $H \cdot \hat{X}$. Bottom row: The same data after multiplication via the GMLVQ relevance matrix $\Omega$.

classes with means $\vec{\mu}_{1}=(-1,0), \vec{\mu}_{2}=(0,0)$ and $\vec{\mu}_{3}=(1,0)$ and shared covariance matrix $\Lambda^{-1}=0.3^{2} \cdot I^{2}$.

The target data is generated with a similar model but with the means set to $\vec{\mu}_{1}=$ $(-0.1,-2), \vec{\mu}_{2}=(0,0)$, and $\vec{\mu}_{3}=(0.1,2)$. Such a rotation of the data is similar to the effect of electrode shifts in the domain of bionic hand prostheses [14]. In both source and target space we generate 100 data points per class.

As a source space model, we employ a GMLVQ model with one prototype per class, which is shown in figure 4 (top left). On the source space data, the GMLVQ model correctly identifies the first dimension as discriminative and discards the second dimension via the relevance matrix $\Omega$ (see figure 4 , bottom left). However, for the target space data this model is invalid because the second dimension now carries the discriminative information (see figure 4, middle column). Quantitatively, we obtain a classification error on the target space data of above $60 \%$ (see figure 5 , left).

For this data set, we only consider the case in which the right class (diamonds in figure 4 is not available in the target space training data. The mean classification error and runtime for this case are shown in figure 5. Even if only four data points are available as training data, the proposed EM transfer learning algorithm consistently identifies a viable transfer mapping $H$ such that the source model achieves a classification error below $1 \%$ (middle). To achieve the same consistency, GMLVQ transfer learning requires at least $2^{6}=64$ data points, while both a-SVM and retrain necessarily fail to classify the missing third class, yielding a classification error of above $30 \%$. These results lend support for $\mathrm{H} 1$ and $\mathrm{H} 2$. 


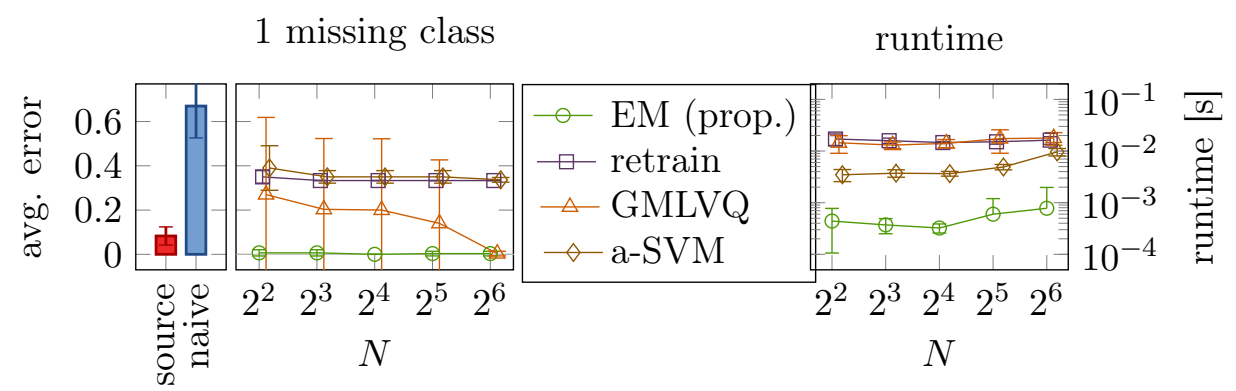

Figure 5: Mean classification error (left, middle) and mean runtime (right) in a ten-fold crossvalidation on the toy data set shown in figure 2 with the left and middle class being available for transfer learning. The $x$-axis indicates the number of available target space training data points $N$ (in log scaling) while the $y$-axis displays the mean classification error (left, middle) or the runtime (right, log scale). Error bars indicate the standard deviation.

Regarding runtime, we observe that our proposed transfer learning scheme is roughly 10 times faster compared to a-SVM and roughly 30 times faster compared to GMLVQ transfer learning and learning a new GMLVQ model (see figure 5, right), supporting H3.

\subsection{Artificial Data II}

Our second artificial data set illustrates the advantage of individual precision matrices in cases where strong class overlap is present. The data set is inspired by the cigars data set by 22 and consists of 1000 data points for each of the three classes. The data is generated via a labeled Gaussian mixture model (lGMM) with one component per class, with means at $\vec{\mu}_{1}=(-0.5,0), \vec{\mu}_{2}=(0.5,0)$, and $\vec{\mu}_{3}=(1.5,0)$, and covariance matrices

$$
\Lambda_{1}^{-1}=\Lambda_{3}^{-1}=\left(\begin{array}{cc}
0.485 & 0.36 \\
0.36 & 0.485
\end{array}\right), \quad \text { and } \quad \Lambda_{2}^{-1}=\left(\begin{array}{cc}
0.485 & -0.36 \\
-0.36 & 0.485
\end{array}\right)
$$

The target data is generated from the same distribution, with the model being rotated by $90^{\circ}$ (see figure 6).

As a source space model, we employ GMLVQ and LGMLVQ with one prototype per class. The challenge in classifying this data lies in the fact that the discriminative direction, that is, the direction orthogonal to the main axis of the classes covariance matrix, is different for the middle class compared to the other two. A lGMM with just one component per class can only account for this difference by using local precision matrices. Accordingly, we observe that the source classification error for GMLVQ (i.e. a IGMM with shared precision matrix) is much higher compared to LGMLVQ (i.e. a lGMM with local precision matrices per component) with $21.33 \%$ versus $9.73 \%$ on average (see source and source-loc in figure 7, left). 

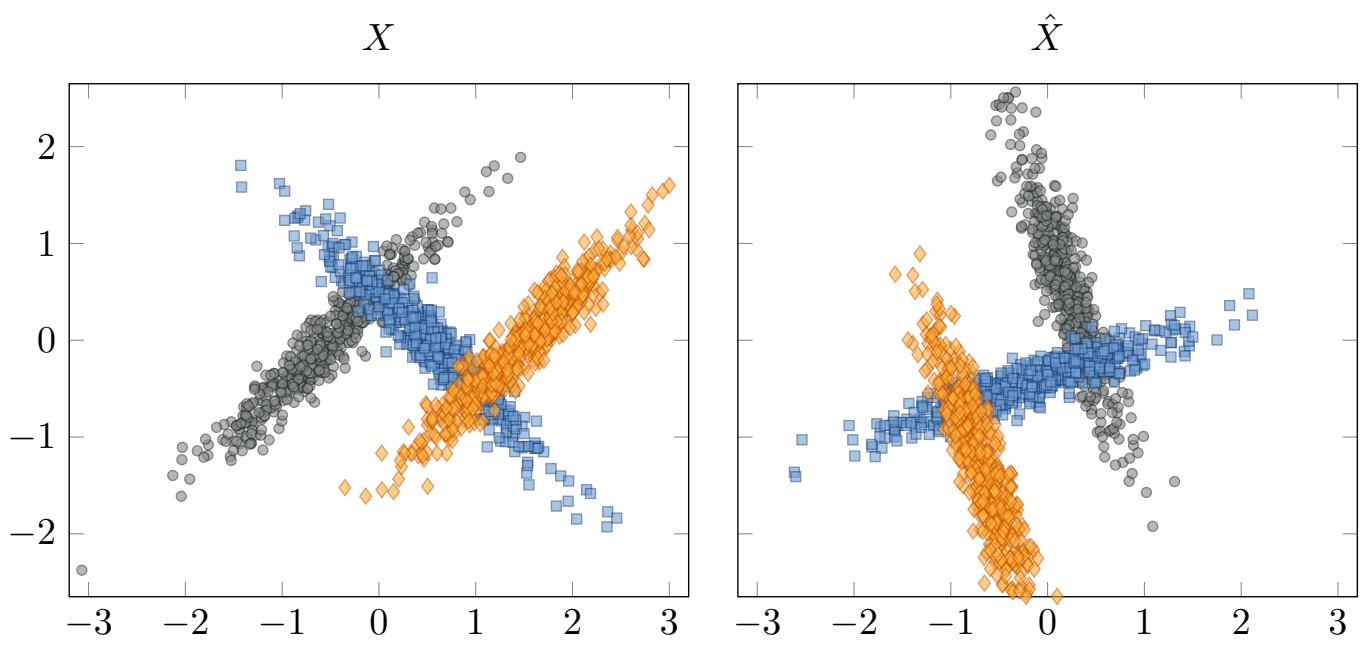

Figure 6: The 3 cigars data set consisting of three classes generated from non-spherical Gaussians. Source data are shown in the left plot and target data in the right.

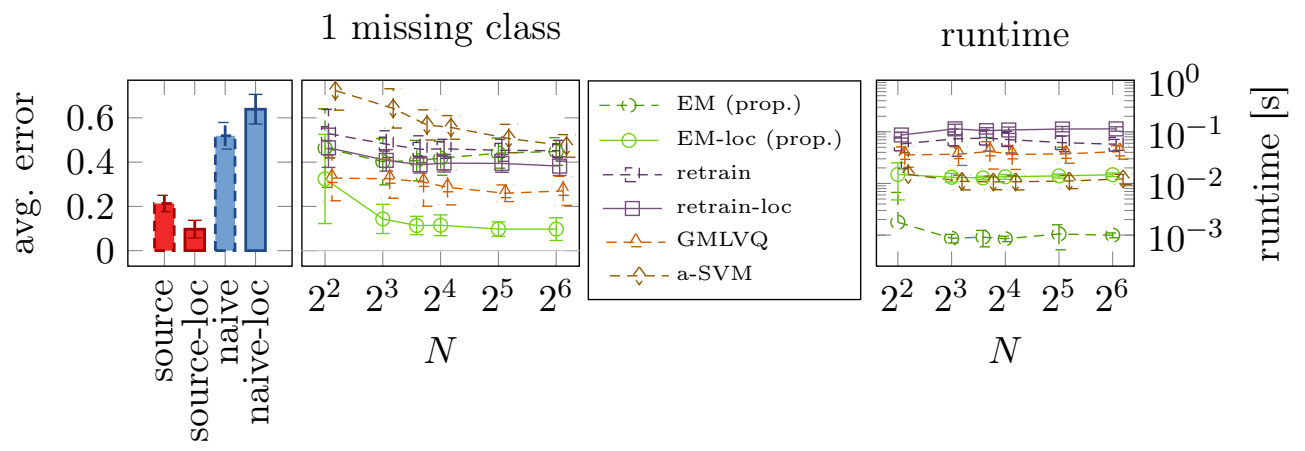

Figure 7: Mean classification error (left, middle) and mean runtime (right) in a 30-fold crossvalidation on the cigars data set shown in figure 6 with the left and middle class being available for transfer learning. The $x$-axis indicates the number of available target space training data points $N$ (in log scaling) while the $y$-axis displays the mean classification (left middle) or the runtime (right, log scale). Error bars indicate the standard deviation. Approaches with individual precision matrices per class are marked with "loc.". Approaches with shared precision matrices are drawn with dashed lines. 
For transfer learning on this data, we utilize only data of the left and middle class. The mean classification error across 30 crossvalidation trials is depicted in figure 7 (middle). As expected, we observe that our proposed transfer learning scheme based on the LGMLVQ model (EM-loc) outperforms all other approaches significantly if at least 12 data points are available $\left(p<10^{-3}\right)$ and achieves an error below $12 \%$ on average, close to the error of the source space model. These results lend support for both $\mathrm{H} 1$ and $\mathrm{H} 2$.

Regarding runtime we observe that the proposed expectation maximization scheme for individual precision matrices (EM-loc) is about 10 times slower compared to the scheme for a shared precision matrix (see figure 7, right). This is due to the fact that the latter approach can exploit a closed-form solution for $H$ while the former needs to employ an iterative solver. This also makes EM-loc about as slow as a-SVM, but still about 3 times faster compared to GMLVQ transfer learning and about 8 times faster compared to retraining a new LGMLVQ model on the target data. Thus, H3 is partially supported.

\subsection{Myoelectric data}

The motivation for our myoelectric data set is to learn a classifier mapping from electromyographic (EMG) recordings to the corresponding hand motion. Such a mapping can be utilized as an user interface for a hand prosthesis because, after amputation, the hand remains represented in the brain. By actuating the so called phantom hand, the residual muscles in the stump are activated via the motor neurons. This leads to corresponding EMG signals that can be mapped to the desired motion, which is then executed by the prosthesis [10.

This data set consists of electromyographic (EMG) recordings of hand motions of 10 able-bodied participants, recorded with a high-density grid of 96 EMG electrodes with 8 $\mathrm{mm}$ inter-electrode distance, located around the forearm at $1 / 3$ of the distance from elbow to wrist.

Each participant performed 15 to 35 runs (236 runs in total) of a series of six hand movements, namely wrist pronation/supination, wrist flexion/extension and finger spread/fist, intermitted by resting phases. Each motion lasted 3 seconds from which the first and the last second were cut to avoid label noise, leaving 1 seconds of each motion for analysis. The experiments are in accordance with the declaration of Helsinki and approved by the local ethics commission. Further details on the experimental protocol are provided in [11].

Our classification task is to identify the correct motion corresponding to the current EMG signal, including an additional resting class (i.e. 7 classes in total). All signals were filtered with a low pass $(500 \mathrm{~Hz}$, fourth-order Butterworth), a high pass $(20 \mathrm{~Hz}$, fourth-order Butterworth), and a band stop filter $(45-55 \mathrm{~Hz}$, second-order Butterworth) to remove noise, movement artifacts, and power line interferences respectively. As features, we employ the logarithm of the signal variance for each electrode, computed on non-overlapping time windows of $100 \mathrm{~ms}$ length. Thus, depending on the number of runs, 1925 to 3255 samples were available per participant, balanced for all classes (for the participant with the fewest runs we obtained 275 samples per class, for the participant with the most runs 465 samples per class).

Since high-density EMG recordings are not common in prosthetic hardware [10], we 


\begin{tabular}{|c|c|c|c|c|c|c|}
\hline$K$ & GMLVQ & LGMLVQ & slGMM & IGMM & $\begin{array}{c}\text { GMLVQ }+ \\
\text { slGMM }\end{array}$ & $\begin{array}{c}\text { LGMLVQ } \\
\text { lGMM }\end{array}$ \\
\hline 1 & $6.7 \pm 7.1 \%$ & $7.0 \pm 7.2 \%$ & $\mathbf{5 . 9} \pm \mathbf{6 . 7} \%$ & $6.7 \pm 7.1 \%$ & $\mathbf{5 . 9} \pm \mathbf{6 . 7} \%$ & $6.7 \pm 7.1 \%$ \\
\hline 2 & $6.5 \pm 6.8 \%$ & $8.4 \pm 7.9 \%$ & $5.8 \pm 6.5 \%$ & $6.5 \pm 6.6 \%$ & $\mathbf{5 . 6} \pm \mathbf{6 . 2} \%$ & $9.9 \pm 8.0 \%$ \\
\hline 3 & $6.7 \pm 7.3 \%$ & $9.3 \pm 8.5 \%$ & $6.1 \pm 6.7 \%$ & $7.1 \pm 7.7 \%$ & $\mathbf{5 . 7} \pm \mathbf{6 . 4} \%$ & $9.6 \pm 8.7 \%$ \\
\hline 4 & $6.5 \pm 7.4 \%$ & $9.9 \pm 8.9 \%$ & $\mathbf{5 . 9} \pm \mathbf{6 . 6} \%$ & $7.8 \pm 7.4 \%$ & $\mathbf{5 . 9} \pm \mathbf{6 . 7} \%$ & $11.9 \pm 12.8 \%$ \\
\hline 5 & $6.4 \pm 7.4 \%$ & $10.1 \pm 8.8 \%$ & $\mathbf{5 . 9} \pm \mathbf{6 . 7} \%$ & $7.8 \pm 7.3 \%$ & $5.9 \pm \mathbf{6 . 4} \%$ & $23.1 \pm 28.9 \%$ \\
\hline
\end{tabular}

Table 1: Mean classification test error and standard deviation on the source space data across all runs in the myoelectric data set. The different classification models are listed on the $\mathrm{x}$ axis, the number of prototypes / Gaussian components for the model on the $\mathrm{y}$ axis. The best results in each row are highlighted via bold print.

simulate a more realistic setup by using a subset of 8 equidistant electrodes located on a ring around the forearm (see figure 1, top left). In order to obtain disturbed target data, we simulate an electrode shift by utilizing eight different electrodes, located one step within the array $(8 \mathrm{~mm})$ transversely to the forearm (see figure 1, bottom left). Such electrode shifts pose a serious problem in real-life prosthesis control, since they occur frequently, e.g. after reapplying the prosthesis, and lead to significantly decreased classification accuracy [10].

As a first analysis, we evaluate which classification method performs best on the source data set. In particular, we compare a generalized matrix learning vector quantization (GMLVQ), local GMLVQ (LGMLVQ), a labeled Gaussian mixture model with shared precision matrix (slGMM), a labeled Gaussian mixture model with individual precision matrices (lGMM), a slGMM with GMLVQ initialization (GMLVQ + slGMM) and a lGMM with LGMLVQ initialization (LGMLVQ + lGMM). The Gaussian mixture models were trained with expectation maximization while restricting the standard deviation in each dimension to be at least 0.001 , as described by [1. For each of the methods, we vary the number of prototypes/Gaussian components from 1 to 5 . In our analysis, we iterate over all 236 runs in the data set and treat the data of the current run as test data, yielding a leave-one-out crossvalidation over the 236 runs. As training data we utilize a random sample of 175 data points, balanced over the classes, drawn from the remaining runs of the same subject. We train each model starting from 5 random initializations and select the model with the lowest training error. For this model, we then record the classification error on the test data.

The results of our pre-experiment are shown in table 1. As can be seen, a labeled Gaussian mixture model with shared precision matrix (slGMM) and GMLVQ initialization consistently achieves the best results. The difference in error is significant compared to GMLVQ $(p<0.05)$, LGMLVQ $(p<0.001)$, IGMM $(p<0.001)$, and IGMM with LGMLVQ initialization $(p<0.001)$. The difference to a slGMM without GMLVQ initialization is insignificant. Regarding the number of prototypes we obtain the best results for $K=2$ prototypes, although the error difference to other values for $K$ is insignificant. For the main analysis, we select the overall best model, namely slGMM with GMLVQ initialization and 


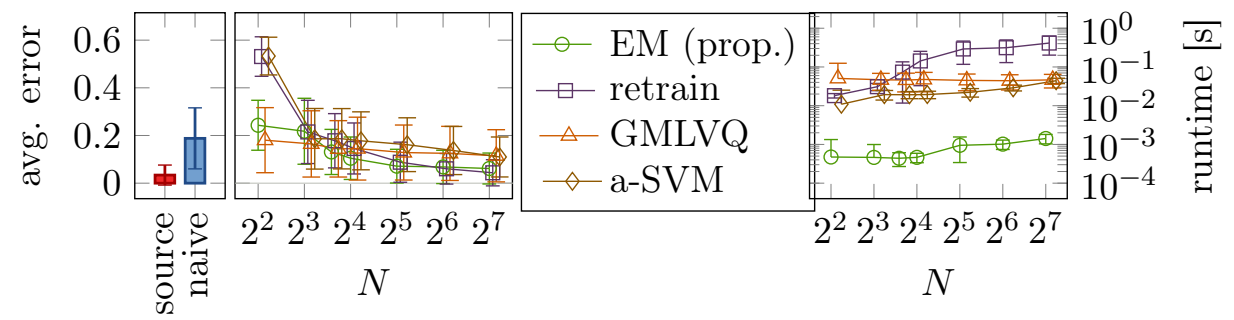

Figure 8: Mean classification error (left, middle) and mean runtime (right) across all runs in the myoelectric data set. The $x$-axis indicates the number of available target space training data points $N$ (in log scaling) while the $y$-axis displays the mean classification error (left, middle) or the runtime (right, log scale). Error bars indicate the standard deviation.

\begin{tabular}{|c|c|c|c|c|c|}
\hline$N$ & naive & EM (prop.) & retrain & GMLVQ & a-SVM \\
\hline 4 & $18.8 \pm 12.8 \%$ & $24.3 \pm 10.5 \%$ & $53.1 \pm 8.3 \%$ & $18.0 \pm 13.6 \%$ & $53.3 \pm 7.9 \%$ \\
\hline 8 & $18.8 \pm 12.8 \%$ & $21.8 \pm 13.8 \%$ & $21.5 \pm 13.3 \%$ & $16.5 \pm 13.9 \%$ & $18.6 \pm 12.7 \%$ \\
\hline 12 & $18.8 \pm 12.8 \%$ & $13.1 \pm \mathbf{9 . 5} \%$ & $17.8 \pm 11.4 \%$ & $14.4 \pm 11.8 \%$ & $18.6 \pm 12.7 \%$ \\
\hline 16 & $18.8 \pm 12.8 \%$ & $10.5 \pm 8.9 \%$ & $14.6 \pm 10.7 \%$ & $14.5 \pm 13.2 \%$ & $17.8 \pm 12.2 \%$ \\
\hline 32 & $18.8 \pm 12.8 \%$ & $7.1 \pm \mathbf{7 . 1} \%$ & $8.8 \pm 8.4 \%$ & $12.9 \pm 11.5 \%$ & $16.1 \pm 11.3 \%$ \\
\hline 64 & $18.8 \pm 12.8 \%$ & $6.8 \pm 7.0 \%$ & $6.0 \pm 6.4 \%$ & $12.5 \pm 11.3 \%$ & $13.7 \pm 10.1 \%$ \\
\hline 128 & $18.8 \pm 12.8 \%$ & $6.2 \pm 6.5 \%$ & $4.4 \pm \mathbf{5 . 5} \%$ & $11.5 \pm 10.9 \%$ & $11.0 \pm 8.4 \%$ \\
\hline
\end{tabular}

Table 2: Mean classification test error and standard deviation across all runs in the myoelectric data set. The different transfer learning approaches are listed on the $\mathrm{x}$ axis, the number of data points $N$ for transfer learning on the y axis. The best results in each row are highlighted via bold print.

$K=2$.

In our main analysis, we first consider the case where data from all classes is available for transfer learning. Again, we iterate over all 236 runs and treat the data in the current run as test data, both for the source as well as for the target space. As training data in the source space, we use the data from all remaining runs of the same subject. We train a slGMM with GMLVQ initialization and $K=2$ starting from 5 random initializations and select the one with the lowest training error. Then, we use a small random sample of the data from all remaining runs of the same subject in the alternative electrode configuration as training data for transfer learning and record the classification error on the (unseen) target space data from the current run.

The mean classification error across all 236 runs is shown in table 2 and figure 8 (left and middle). Several significant effects can be observed using a one-sided Wilcoxon signed rank test:

1. Classification performance degrades if an electrode shift is applied, i.e. the naive error is significantly higher than the source error $\left(p<10^{-3}\right)$. 


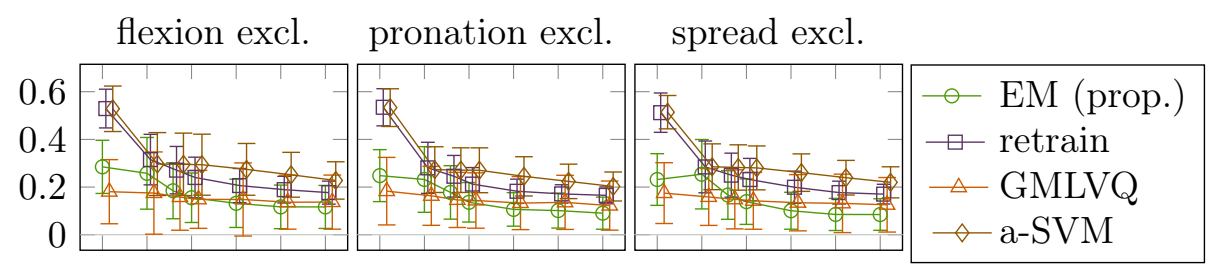

extension excl. supination excl. fist excl.

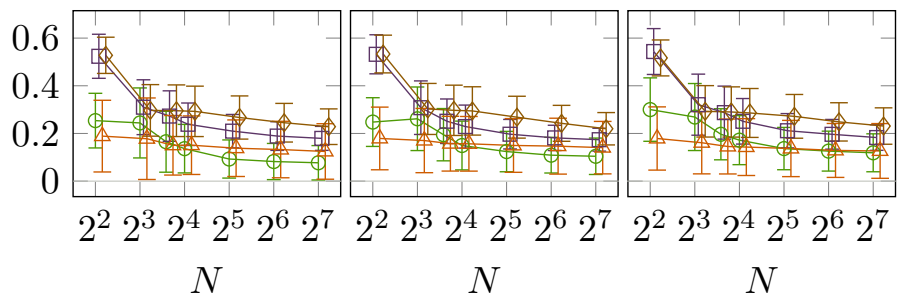

Figure 9: Mean classification error across all runs in the myoelectric data set if one movement was excluded from the training data for transfer learning. The excluded class is listed in the title of each plot. The $x$-axis indicates the number of available target space training data points $N$ (in log scaling) while the $y$-axis displays the mean classification error. Error bars indicate the standard deviation.

2. If at least 12 data points are available for training, our proposed algorithm outperforms a naive application of the source space model $\left(p<10^{-3}\right)$.

3. If between 12 and 32 data points are available, the proposed scheme outperforms a retrained model on the target data $\left(p<10^{-3}\right)$, lending support for H1.

4. If at least 12 data points are available for training, our proposed algorithm outperforms the adaptive SVM $\left(p<10^{-3}\right)$.

5. If at least 16 data points are available for training, our proposed algorithm outperforms gradient-based learning on the GMLVQ cost function $\left(p<10^{-3}\right)$.

With regards to runtime, we note that our proposed algorithm is roughly 30 times faster compared to GMLVQ and a-SVM and roughly 100 times faster compared to re-training a new model on the target space data (see figure 8, right), supporting H3.

With regards to $\mathrm{H} 2$, we consider the case of single motions missing from the training data for transfer learning. This is motivated by the practical application scenario of bionic prostheses. In practice, as soon as a user notes deteriorating classification accuracy of her hand prosthesis, she would have to record new labeled training data to learn a transfer mapping which enhances accuracy again. In this recording process, the user would have to execute a precisely timed calibration sequence of neural patterns which correspond to desired hand motions. Any mistakes in timing introduce label noise into the training data and thus may lead to deteriorating performance. Therefore, any motion which has not to be recorded reduces the likelihood of label noise and enhances ease-of-use. 


\begin{tabular}{|c|c|c|c|c|c|}
\hline$N$ & naive & EM (prop.) & retrain & GMLVQ & a-SVM \\
\hline 4 & $18.8 \pm 12.8 \%$ & $25.4 \pm 11.5 \%$ & $52.4 \pm 9.2 \%$ & $18.9 \pm 15.0 \%$ & $52.8 \pm 7.6 \%$ \\
\hline 8 & $18.8 \pm 12.8 \%$ & $24.4 \pm 14.7 \%$ & $31.0 \pm 11.5 \%$ & $17.8 \pm 17.1 \%$ & $29.7 \pm 10.8 \%$ \\
\hline 12 & $18.8 \pm 12.8 \%$ & $16.5 \pm 12.8 \%$ & $27.3 \pm 10.6 \%$ & $15.0 \pm 12.5 \%$ & $29.5 \pm 10.8 \%$ \\
\hline 16 & $18.8 \pm 12.8 \%$ & $13.6 \pm 10.2 \%$ & $23.9 \pm 8.9 \%$ & $15.1 \pm 12.3 \%$ & $29.3 \pm 10.6 \%$ \\
\hline 32 & $18.8 \pm 12.8 \%$ & $9.3 \pm 8.0 \%$ & $21.0 \pm 7.0 \%$ & $13.8 \pm 11.9 \%$ & $26.7 \pm 9.1 \%$ \\
\hline 64 & $18.8 \pm 12.8 \%$ & $8.2 \pm 7.6 \%$ & $18.9 \pm 6.0 \%$ & $13.3 \pm 11.8 \%$ & $24.5 \pm 8.1 \%$ \\
\hline 128 & $18.8 \pm 12.8 \%$ & $7.7 \pm 7.2 \%$ & $17.9 \pm 5.0 \%$ & $12.4 \pm 11.6 \%$ & $22.8 \pm 7.5 \%$ \\
\hline
\end{tabular}

Table 3: Mean classification test error and standard deviation across all runs in the myoelectric data set when no samples for the extension movement were available for transfer learning. The different transfer learning approaches are listed on the $\mathrm{x}$ axis, the number of data points $N$ for transfer learning on the y axis. The best results in each row are highlighted via bold print.

We repeated our experiments with each of the six motions being missing from the training data for transfer learning (the resting class can be considered easy to record because users do not have to actively produce any specific neural pattern). We also experimented with omitting more than one class in the training data, but observed that under these conditions no transfer method outperformed the baseline of naively applying the source model to the target space data.

The average results across participants and trials are depicted in table 3 and figure 9 . Several significant effects can be observed using a one-sided Wilcoxon signed rank test:

1. If at least 32 data points are available for training, our proposed algorithm outperforms a naive application of the source space model $\left(p<10^{-3}\right)$.

2. Irrespective of the number of available data points, our EM transfer learning scheme outperforms a retrained model on the target data $\left(p<10^{-3}\right)$.

3. If at least 12 data points are available for training, our proposed algorithm outperforms the adaptive SVM $\left(p<10^{-3}\right)$.

4. If extension, pronation, supination, or spread are excluded and at 32 data points are available for training, our proposed algorithm outperforms gradient-based learning on the GMLVQ cost function $(p<0.01)$.

In conjunction, these results support $\mathrm{H} 2$.

\section{Conclusion}

We have presented a novel expectation maximization (EM) algorithm which learns a linear transfer function between a target and a source space. This transfer function maximizes the likelihood of target space data according to a labeled Gaussian mixture model in the source space. We demonstrated how this algorithm can be combined with models from the 
learning vector quantization family, in particular generalized learning vector quantization (GMLVQ) and local GMLVQ. We have argued that learning a transfer function between target and source space is easier compared to re-learning a full classification model in the target space, if the change in representation between both spaces is structurally simple, that is, approximately linear.

In our experiments, we evaluated our approach on two artificial data sets and a data set of real electromyographic (EMG) data from the domain of bionic hand prostheses. In all cases, our proposed method was able to learn a transfer mapping which significantly improved the classification accuracy compared to all baselines, in particular if few target space samples were available and a class was not represented in the training set. The latter aspect is particularly relevant in settings where recording data for an additional class may be demanding, as is the case in bionic hand prostheses.

Overall, our proposed transfer learning approach appears as a simple, data- and timesaving alternative compared to re-learning a new classification model, and even other domain adaptation and transfer learning approaches. For future work, it may be interesting to explore whether the learned transfer function can be used to transfer other classification models than those from the learning vector quantization family, whether the transfer function can be adjusted on-line, and whether theoretic guarantees for transfer learning are possible. Beyond these machine learning questions we hope to use transfer learning to get one step closer towards an intuitive, rapid, and robust user interface for bionic prostheses that helps amputees to achieve better hand function in everyday tasks.

\section{Acknowledgement}

Funding by the DFG under grant number HA 2719/6-2, the CITEC center of excellence (EXC 277), and the EU-Project "Input" (grant number 687795) is gratefully acknowledged. We also thank our reviewers for their insightful comments which helped to improve the quality of our contribution.

\section{References}

[1] D. Barber. Bayesian Reasoning and Machine Learning. Cambridge University Press, Cambridge, UK, 2012. URL: http://web4.cs.ucl.ac.uk/staff/D.Barber/pmwiki/ pmwiki.php?n=Brml. HomePage.

[2] J. T. Belter, J. L. Segil, A. M. Dollar, and R. F. Weir. Mechanical design and performance specifications of anthropomorphic prosthetic hands: A review. Journal of Rehabilitation Research \& Development, 50(5):599-618, 2013. doi:10.1682/JRRD. 2011.10.0188.

[3] S. Ben-David, J. Blitzer, K. Crammer, and F. Pereira. Analysis of representations for domain adaptation. In T. H. Bernhard Schölkopf, John C. Platt, editor, Proceedings of the 19th Advances in Neural Information Processing Systems Confer- 
ence (NIPS 2006), pages 137-144, 2006. URL: https://papers.nips.cc/paper/ 2983-analysis-of-representations-for-domain-adaptation.

[4] C. M. Bishop. Pattern Recognition and Machine Learning. Springer, Secaucus, NJ, USA, 2006.

[5] P. Blöbaum, A. Schulz, and B. Hammer. Unsupervised Dimensionality Reduction for Transfer Learning. In M. Verleysen, editor, Proceedings of the 23rd European Symposium on Artificial Neural Networks, Computational Intelligence and Machine Learning, ESANN 2015, Bruges, Belgium, April, 22-24, 2015, pages 507-512. i6doc.com, 2015.

[6] C. Cortes, M. Mohri, M. Riley, and A. Rostamizadeh. Sample selection bias correction theory. In Y. Freund, L. Györfi, G. Turán, and T. Zeugmann, editors, Proceedings of the 19th International Conference on Algorithmic Learning Theory (ALT 2008), pages 38-53, Budapest, Hungary, 2008. Springer. doi:10.1007/978-3-540-87987-9_8.

[7] A. P. Dempster, N. M. Laird, and D. B. Rubin. Maximum likelihood from incomplete data via the EM algorithm. Journal of the Royal Statistical Society. Series B, 39(1):138, 1977.

[8] G. Ditzler, M. Roveri, C. Alippi, and R. Polikar. Learning in nonstationary environments: A survey. IEEE Computational Intelligence Magazine, 10(4):12-25, 2015. doi:10.1109/MCI.2015.2471196.

[9] P. L. Fackler. Notes on matrix calculus. Technical report, North Carolina State University, 2005. URL: http://www4.ncsu.edu/ pfackler/MatCalc.pdf.

[10] D. Farina, N. Jiang, H. Rehbaum, A. Holobar, B. Graimann, H. Dietl, and O. C. Aszmann. The extraction of neural information from the surface emg for the control of upper-limb prostheses: Emerging avenues and challenges. IEEE Transactions on Neural Systems and Rehabilitation Engineering, 22(4):797-809, July 2014. doi:10. 1109/TNSRE. 2014.2305111.

[11] J. M. Hahne, B. Graimann, and K. R. Müller. Spatial filtering for robust myoelectric control. IEEE Transactions on Biomedical Engineering, 59(5):1436-1443, May 2012. doi:10.1109/TBME.2012.2188799.

[12] J. Huang, A. Gretton, K. M. Borgwardt, B. Schölkopf, and A. J. Smola. Correcting sample selection bias by unlabeled data. In T. H. Bernhard Schölkopf, John C. Platt, editor, Proceedings of the 19th Advances in Neural Information Processing Systems Conference (NIPS 2006), pages 601-608. MIT Press, 2007. URL: http://papers.nips.cc/paper/ 3075-correcting-sample-selection-bias-by-unlabeled-data.pdf.

[13] Y. Huang, K. B. Englehart, B. Hudgins, and A. D. C. Chan. A gaussian mixture model based classification scheme for myoelectric control of powered upper limb 
prostheses. IEEE Transactions on Biomedical Engineering, 52(11):1801-1811, 2005. doi:10.1109/TBME.2005.856295.

[14] R. N. Khushaba, M. Takruri, J. V. Miro, and S. Kodagoda. Towards limb position invariant myoelectric pattern recognition using time-dependent spectral features. Neural Networks, 55:42-58, 2014. doi:10.1016/j.neunet.2014.03.010.

[15] T. Kohonen. Learning Vector Quantization, pages 175-189. Springer, Berlin/Heidelberg, Germany, 1995. doi:10.1007/978-3-642-97610-0_6.

[16] V. Losing, B. Hammer, and H. Wersing. Knn classifier with self adjusting memory for heterogeneous concept drift. In Proceedings of the 16th IEEE International Conference on Data Mining (ICDM 2016), pages 291-300, 2016. doi:10.1109/ICDM.2016.0040.

[17] B. Paaßen, A. Schulz, and B. Hammer. Linear Supervised Transfer Learning for Generalized Matrix LVQ. In B. Hammer, T. Martinetz, and T. Villmann, editors, Proceedings of the Workshop New Challenges in Neural Computation (NC2) 2016, number 4, pages 11-18, Hannover, Germany, 2016.

[18] B. Paaßen and A. Schulz. Linear Supervised Transfer Learning Toolbox, 2017. Version 0.2.2. doi:10.4119/unibi/2912671.

[19] S. J. Pan and Q. Yang. A survey on transfer learning. IEEE Transactions on Knowledge and Data Engineering, 22(10):1345-1359, Oct 2010. doi:10.1109/TKDE.2009. 191 .

[20] C. Prahm, B. Paaßen, A. Schulz, B. Hammer, and O. Aszmann. Transfer learning for rapid re-calibration of a myoelectric prosthesis after electrode shift. In J. Ibáñez, J. González-Vargas, J. M. Azorín, M. Akay, and J. L. Pons, editors, Converging Clinical and Engineering Research on Neurorehabilitation II: Proceedings of the 3rd International Conference on NeuroRehabilitation (ICNR2016), October 1821, 2016, Segovia, Spain, pages 153-157. Springer International Publishing, 2016. doi:10.1007/978-3-319-46669-9_28.

[21] S. Saralajew and T. Villmann. Transfer learning in classification based on manifold models and its relation to tangent metric learning. In C. J. Yoonsuck Choe, editor, Proceedings of the 2017 International Joint Conference on Neural Networks (IJCNN 2017), Anchorage, AK, USA, 2017. in press.

[22] P. Schneider, M. Biehl, and B. Hammer. Adaptive relevance matrices in learning vector quantization. Neural Computation, 21(12):3532-3561, 2009. doi:10.1162/ neco.2009.11-08-908.

[23] P. Schneider, M. Biehl, and B. Hammer. Distance learning in discriminative vector quantization. Neural Computation, 21(10):2942-2969, 2009. doi:10.1162/neco. 2009.10-08-892. 
[24] S. Seo and K. Obermayer. Soft learning vector quantization. Neural Computation, 15(7):1589-1604, 2003. doi:10.1162/089976603321891819.

[25] H. Shimodaira. Improving predictive inference under covariate shift by weighting the log-likelihood function. Journal of statistical planning and inference, 90(2):227-244, 2000 .

[26] J. Yang, R. Yan, and A. G. Hauptmann. Cross-domain video concept detection using adaptive SVMs. In Proceedings of the 15th ACM International Conference on Multimedia (MM '07), pages 188-197. ACM, 2007. doi:10.1145/1291233.1291276. 